\title{
Bacteriemia por Raoultella planticola: descripción de un caso y revisión de la literatura
}

\author{
Raoultella planticola bacteraemia: a case report and review of the literature
}

\author{
Sílvia Pacheco de Sousa ${ }^{1}$, Ana Filipa Teixeira Ferreira'1, Maria Margarida Luís², Mari Mesquita ${ }^{3}$ \\ 'Interna de Formação Geral, Serviço de Medicina Interna. ${ }^{2}$ Assistente Hospitalar, Serviço de Medicina Interna. ${ }^{3}$ Assistente Hospitalar Graduada Sénior, Diretora de Serviço, Serviço de Medicina Interna. Centro Hospitalar do \\ Tâmega e Sousa, Portugal
}

\begin{abstract}
Raoultella planticola (R. planticola) is an anaerobic gram-negative bacillus implicated in urinary, intra-abdominal, skin and soft tissue infections, pneumonia and bacteraemia. We depict here the clinical case of a 74 -year-old woman, medicated lifelong with phenytoin, with bacteraemia caused by $R$. planticola, successfully treated with ceftriaxone. To date, a comprehensive literature review, revealed 52 published clinical cases (between 20072019), thirteen of which due to bacteraemia.

Keywords: Raoultella planticola, bacteraemia.
\end{abstract}

\section{INTRODUCTION}

Raoultella planticola ( $R$. planticola) is an immobile, encapsulated, anaerobic gram-negative bacillus, belonging to the Enterobacteriaceae family, genus Klebsiella ${ }^{1-3}$. Increasingly recognized as a clinically significant entity, $R$. planticola is often implicated in urinary, intra-abdominal, skin and soft tissue infections, as well as pneumonia and bacteraemia ${ }^{4-6}$. In this article we describe another case of $R$. planticola bacteraemia.

\section{CASE REPORT}

We report a case of a 74-year-old fragile woman with low functional status (Katz 2/6), with known comorbidities of dementia, epilepsy (chronically medicated with phenytoin), obesity, hypertension, dyslipidemia, colonic diverticular disease and a previous history of alcohol abuse (accordingly to family, abstinent for 7 years). Patient was admitted to the emergency department due to generalized tonic-clonic seizures, witnessed by her family, during mealtime. The patient presented signs of respiratory distress and depressed level of consciousness (Glasgow coma scale 8) but hemodynamically stable and afebrile. A fragment of meat was identified in the oropharynx and removed. Patient evaluation performed at admission revealed: chest $X$-ray with a heterogeneous hypotransparency in the right lower lobe and cranial computed tomography with no signs of acute vascular lesions or space-occupying lesions. Laboratory tests revealed increased inflammatory parameters, signs rhabdomyolysis, acute kidney injury (AKI) (serum creatinine $1.91 \mathrm{mg} / \mathrm{dL}$ ), and infratherapeutic levels of phenytoin. Arterial blood gas showed hypoxemic respiratory failure. Thus, patient was admitted in the Internal Medicine ward under the assumption of diagnosis of pneumonitis/ aspiration pneumonia. Prior to initiation of empirical antimicrobial therapy with amoxicillin/clavulanate, blood cultures were obtained. The first days after hospitalization, patient was febrile and presented a slight degree of prostration, with no focal neurological deficits. No cardiac murmurs, palpable adenomegalies, organomegaly or masses, as well as changes in the integument were identified. AKI progressed to a maximum creatinine value of $6.63 \mathrm{mg} / \mathrm{dL}$ and gradually resolved to previous values. On the fifth day of hospitalization, blood cultures were positive for gram-negative bacillus, and antibiotic therapy was escalated to piperacillin/tazobactam.

\section{RESUMEN}

Raoultella planticola (R. planticola) es un bacilo anaerobio Gram negativo implicado en infecciones de partes blandas, urinarias, intra-abdominales, neumonías y episodios de bacteriemia. Presentamos el caso de una mujer de 74 años, tratada con fenitoína de forma crónica, con bacteriemia por R. planticola tratada de forma exitosa con ceftriaxona. Hasta la fecha, tras realizar una revisión de la literatura, se han descrito 52 casos entre 2007 y 2019, trece de los cuales presentaron bacteriemia.

Palabras clave: Raoultella planticola, bacteriemia.

One day later, $R$. planticola was isolated in one of the blood cultures, resistant to ampicillin and sensitive to gentamicin, amoxicillin/ clavulanate, cefuroxime sodium and clotrimazole. In this context, antimicrobial therapy was adapted to ceftriaxone, during the next 7 days. Follow-up blood cultures persisted sterile. Echocardiography was requested, which excluded vegetations, destructive lesions and abscess formation. Complete blood count and clinical chemistry showed normocytic and normochromic anemia with stable hemoglobin $(10 \mathrm{~g} / \mathrm{dL})$ paired with iron and B12 deficiencies, promptly treated (no blood loss documented); serum proteinogram showed no relevant deviations, no hypercalcemia, normal thyroid-stimulating hormone, non-reactive serologies for human immunodeficiency virus and hepatitis $B$ and $C$, non-reactive VDRL, normal angiotensin converting enzyme, a negative summary autoimmune panel with increased immunoglobulin (lg) A and lgG (508 and 1753 mg/ $\mathrm{dL}$, respectively) and increased erythrocyte sedimentation rate (97 $\mathrm{mm} / \mathrm{h})$. Mycobacterium tuberculosis infection was excluded. Chest, abdomen and pelvis computed tomography found signs of recent pulmonary thromboembolism at the level of the left upper and lower lobar branch, right interlobar branch and right segmental branches of the lower lobe, associated with signs of thrombosis of the portal vein, in a context of slightly lobulated contours enlarged liver. Anticoagulant treatment with apixaban was decided.

Progressive clinical improvement was observed during hospitalization, registering a full recovery at discharge.

\section{LITERATURE REVIEW AND DISCUSSION}

R. planticola, previously known as Klebsiella planticola and Klebsiella trevisanii, is a bacterium found in water and soil. Despite of its increasing clinical relevance, it remains a rare cause of infection in humans ${ }^{1-3}$.

It is thought to have a virulence capacity similar to that of Klebsiella pneumoniae ${ }^{7}$, presenting with lipopolysaccharides, fimbriae, toxins and biofilm formation ${ }^{8}$, and it is estimated that approximately 9 to $18 \%$ of humans are colonized by $R$. planticola 4 .

Most cases of infection are associated with significant comorbidities, invasive procedures or previous trauma ${ }^{9}$, but the main 


\begin{tabular}{|c|c|c|c|c|c|c|c|}
\hline Author & Year & $\begin{array}{c}\text { Clinical } \\
\text { manifestation }\end{array}$ & Culture site & Risk factors & Age/Gender & Treatment & Outcome \\
\hline $\begin{array}{l}\text { Pacilli, M.; } \\
\text { Nataraja R.M. }\end{array}$ & 2019 & Peritonitis & $\begin{array}{l}\text { Peritoneal } \\
\text { fluid }\end{array}$ & Meckel diverticulum & $\begin{array}{l}8 \text { years/ } \\
\text { Female }\end{array}$ & $\begin{array}{c}\text { Trimethoprim- } \\
\text { sulfamethoxazole (10 } \\
\text { days) }\end{array}$ & $\begin{array}{c}\text { Stable at } 6 \\
\text { months of follow- } \\
\text { up }\end{array}$ \\
\hline $\begin{array}{l}\text { Branco, J. C. } \\
\text { et al }\end{array}$ & 2019 & Peritonitis & $\begin{array}{l}\text { Peritoneal } \\
\text { fluid }\end{array}$ & $\begin{array}{l}\text { Alcoholic liver cirrhosis, } \\
\text { diabetes mellitus (DM) }\end{array}$ & $\begin{array}{l}76 \text { years/ } \\
\text { Male }\end{array}$ & Ceftriaxone (7 days) & Deceased \\
\hline $\begin{array}{l}\text { Harmon, S.L; } \\
\text { Nadeem I. }\end{array}$ & 2019 & Cystitis & Urine & Transplant, DM & $\begin{array}{l}40 \text { years/ } \\
\text { Female }\end{array}$ & $\begin{array}{l}\text { Amoxicillin/clavulanate } \\
\text { (5 days) }\end{array}$ & $\begin{array}{c}3 \text { month following } \\
\text { treatment new } \\
\text { RP infection }\end{array}$ \\
\hline $\begin{array}{l}\text { Fager, C.; } \\
\text { Yurteri-Kaplan, } \\
\text { L }\end{array}$ & 2019 & Cystitis & Urine & $\begin{array}{l}\text { Postoperative, peripheral } \\
\text { neuropathy }\end{array}$ & $\begin{array}{l}50 \text { years/ } \\
\text { Female }\end{array}$ & Nitrofurantoin & $\begin{array}{l}\text { Stable at } 1 \\
\text { month of follow- } \\
\text { up }\end{array}$ \\
\hline $\begin{array}{l}\text { AlSweed A. } \\
\text { et al }\end{array}$ & 2018 & Endocarditis & Blood & $\begin{array}{l}\text { Gastroesophageal reflux } \\
\text { disease (GERD), hiatus } \\
\text { hernia, methicillin- } \\
\text { resistant Staphylococcus } \\
\text { aureus (MRSA) }\end{array}$ & 4 years/Male & $\begin{array}{c}\text { Initially vancomycin } \\
\text { + ceftazidime. } \\
\text { After antimicrobial } \\
\text { susceptibility testing } \\
\text { ceftriaxone + gentamicin }\end{array}$ & $?$ \\
\hline $\begin{array}{l}\text { Al-Sawaf, } 0 . \\
\text { et al }\end{array}$ & 2019 & Cellulitis & Blood & Cancer & $\begin{array}{l}38 \text { years/ } \\
\text { Male }\end{array}$ & $\begin{array}{c}\text { Piperacillin/tazobactam } \\
\text { (12 days) }\end{array}$ & $?$ \\
\hline $\begin{array}{l}\text { Fager C.; } \\
\text { Yurteri-Kaplan } \\
\text { L. }\end{array}$ & 2018 & Cystitis & Urine & $\begin{array}{l}\text { Lupus treated with } \\
\text { methotrexate, postoperative }\end{array}$ & $\begin{array}{l}50 \text { years/ } \\
\text { Female }\end{array}$ & Nitrofurantoin & $\begin{array}{l}\text { Stable at } 1 \\
\text { month of follow- } \\
\text { up }\end{array}$ \\
\hline $\begin{array}{l}\text { Gonzales } \\
\text { Zamora, J.A. } \\
\text { et al }\end{array}$ & 2018 & Bacteremia & Blood & Burns & $\begin{array}{l}85 \text { years/ } \\
\text { Female }\end{array}$ & $\begin{array}{c}\text { Initially Cefepime. } \\
\text { After antimicrobial } \\
\text { susceptibility testing } \\
\text { ceftazidime/avibactam } \\
\text { + polymyxin } \\
\text { (15 days) }\end{array}$ & $?$ \\
\hline $\begin{array}{l}\text { Mehmood H. } \\
\text { et al }\end{array}$ & 2018 & Pyelonephritis & Urine & $\begin{array}{l}\text { Immunoglobulin A } \\
\text { nephropathy }\end{array}$ & $\begin{array}{l}65 \text { years/ } \\
\text { Male }\end{array}$ & $\begin{array}{l}\text { During hospitalization } \\
\text { ceftriaxone ( } 3 \text { days). After } \\
\text { discharge ciprofloxacin } \\
\text { (4 days) }\end{array}$ & $\begin{array}{c}\text { Stable at } 2 \\
\text { weeks of follow- } \\
\text { up }\end{array}$ \\
\hline $\begin{array}{l}\text { Yumoto, T. } \\
\text { et al }\end{array}$ & 2018 & Bacteremia & Blood & Burns & $\begin{array}{l}79 \text { years/ } \\
\text { Male }\end{array}$ & $\begin{array}{c}\text { Initially } \\
\text { piperacillin/tazobactam (9 } \\
\text { days), then Meropenem } \\
\text { (3 days) }\end{array}$ & Deceased \\
\hline $\begin{array}{l}\text { Naganathan, } \\
\text { G.; Amin, N.K. }\end{array}$ & 2018 & $\begin{array}{l}\text { Necrotizing } \\
\text { appendicitis }\end{array}$ & Blood & $\begin{array}{l}\text { Colitis, GERD, proton-pump } \\
\text { inhibitor (PPI), asthma, } \\
\text { postoperative }\end{array}$ & $\begin{array}{l}63 \text { years/ } \\
\text { Female }\end{array}$ & $\begin{array}{l}\text { Initially ceftriaxone (1 } \\
\text { day). Then } \\
\text { amoxicillin/clavulanate } \\
\text { (14 days) }\end{array}$ & $\begin{array}{c}\text { Stable at } 5 \\
\text { weeks of follow- } \\
\text { up }\end{array}$ \\
\hline $\begin{array}{l}\text { Yamamoto, S. } \\
\text { et al }\end{array}$ & 2018 & Bacteremia & Blood & Cancer & $\begin{array}{l}81 \text { years/ } \\
\text { Male }\end{array}$ & $\begin{array}{l}\text { Initially ampicillin } 3 \\
\text { days. After antimicrobial } \\
\text { susceptibility testing, } \\
\text { ceftriaxone (11 days) }\end{array}$ & $?$ \\
\hline Atici, S. et al & 2018 & Bacteremia & Blood & Pre-term newborn & $\begin{array}{c}\text { Newborn with } \\
28 \text { weeks of } \\
\text { gestation }\end{array}$ & $\begin{array}{l}\text { Initially netilmicin, then } \\
\text { piperacillin/tazobactam }\end{array}$ & Deceased \\
\hline $\begin{array}{l}\text { Kalaria, S.S. } \\
\text { et al }\end{array}$ & 2017 & Skin infection & Pus & Trauma (animal bite), PPI & $\begin{array}{l}73 \text { years/ } \\
\text { Female }\end{array}$ & Levofloxacin & $\begin{array}{c}\text { Stable at } 4 \\
\text { months of follow- } \\
\text { up }\end{array}$ \\
\hline $\begin{array}{l}\text { Casarsa, C., } \\
\text { Mearelli, F., } \\
\text { Biolo, G. }\end{array}$ & 2017 & Bacteremia & Blood & $\begin{array}{c}\text { Sjögren syndrome, cancer, } \\
\text { post endoscopic retrograde } \\
\text { cholangiopancreatography } \\
\text { (ERCP) }\end{array}$ & $\begin{array}{l}55 \text { years/ } \\
\text { Female }\end{array}$ & Ceftriaxone (14 days) & $?$ \\
\hline $\begin{array}{l}\text { Yoshida, N.; } \\
\text { Tsuchida, Y. }\end{array}$ & 2017 & $\begin{array}{l}\text { Palmar } \\
\text { atheroma } \\
\text { infection }\end{array}$ & Pus & Cancer & $\begin{array}{l}74 \text { years/ } \\
\text { Male }\end{array}$ & $\begin{array}{l}\text { Initially ceftriaxone (4 } \\
\text { days), then levofloxacin } \\
\text { (21 days) }\end{array}$ & $?$ \\
\hline
\end{tabular}




\begin{tabular}{|c|c|c|c|c|c|c|c|}
\hline Author & Year & $\begin{array}{c}\text { Clinical } \\
\text { manifestation }\end{array}$ & Culture site & Risk factors & Age/Gender & Treatment & Outcome \\
\hline $\begin{array}{l}\text { Povlow, M.R.; } \\
\text { Carrizosa, J.; } \\
\text { Jones, A. }\end{array}$ & 2017 & Bacteremia & Blood & $\begin{array}{l}\text { Alcoholic liver cirrhosis, } \\
\text { seafood consumption }\end{array}$ & $\begin{array}{l}66 \text { years/ } \\
\text { Male }\end{array}$ & $\begin{array}{l}\text { Initially piperacillin/ } \\
\text { tazobactam. After } \\
\text { antimicrobial } \\
\text { susceptibility testing, } \\
\text { ceftriaxone }\end{array}$ & $?$ \\
\hline Subedi, R. et al & 2017 & Bacteremia & Blood & $\begin{array}{l}\text { DM, hypertension, } \\
\text { dyslipidemia }\end{array}$ & $\begin{array}{l}63 \text { years/ } \\
\text { Male }\end{array}$ & $\begin{array}{l}\text { Piperacillin/tazobactam } \\
\text { for } 6 \text { weeks }+ \\
\text { levofloxacin for } 2 \text { weeks }\end{array}$ & Stable \\
\hline $\begin{array}{l}\text { Ulkent, S.C. } \\
\text { et al }\end{array}$ & 2017 & Cholecystitis & Pus & No relevant medical history & $\begin{array}{l}71 \text { years/ } \\
\text { Female }\end{array}$ & Ampicillin (10 days) & $\begin{array}{c}\text { Stable at } 3 \\
\text { months of follow- } \\
\text { up }\end{array}$ \\
\hline $\begin{array}{l}\text { Howell, C.; } \\
\text { Fakhoury, J. }\end{array}$ & 2017 & Cystitis & Urine & Neonatal hyperbilirrubinemia & $\begin{array}{l}2 \text { months/ } \\
\text { Female }\end{array}$ & $\begin{array}{c}\text { Initially ceftriaxone (1 } \\
\text { dose), then cefalexin (10 } \\
\text { days) }\end{array}$ & $?$ \\
\hline $\begin{array}{l}\text { Westerveld, D. } \\
\text { et al }\end{array}$ & 2017 & Pneumonia & Sputum & Cancer & $\begin{array}{l}36 \text { years/ } \\
\text { Female }\end{array}$ & $\begin{array}{l}\text { Initially ceftriaxone, after } \\
\text { exacerbation piperacillin/ } \\
\text { tazobactam }\end{array}$ & $?$ \\
\hline $\begin{array}{l}\text { Bardellini, E. } \\
\text { et al }\end{array}$ & 2017 & Mucositis & Pus & Cancer & $\begin{array}{l}16 \text { years/ } \\
\text { Male }\end{array}$ & $\begin{array}{c}\text { Amikacin + Ceftazidime } \\
\text { (8 days) }\end{array}$ & $\begin{array}{c}\text { Stable at } 12 \\
\text { months of follow- } \\
\text { up }\end{array}$ \\
\hline $\begin{array}{l}\text { Adjodah, C. } \\
\text { et al }\end{array}$ & 2017 & $\begin{array}{l}\text { Cardiac device } \\
\text { implantation site }\end{array}$ & Pus & $\begin{array}{l}\text { Rheumatoid arthritis treated } \\
\text { with methotrexate }\end{array}$ & $\begin{array}{l}79 \text { years/ } \\
\text { Male }\end{array}$ & $\begin{array}{c}\text { Initially oxacillin. } \\
\text { After antimicrobial } \\
\text { susceptibility testing } \\
\text { ciprofloxacin (15 days) }\end{array}$ & $\begin{array}{c}\text { Stable at } 5 \text { years } \\
\text { of follow-up }\end{array}$ \\
\hline Bonnet, E. et al & 2017 & Joint infection & $\begin{array}{l}\text { Synovial } \\
\text { fluid }\end{array}$ & Arthroscopy & $\begin{array}{l}46 \text { years/ } \\
\text { Male }\end{array}$ & $\begin{array}{c}\text { Initially vancomycin. } \\
\text { After antimicrobial } \\
\text { susceptibility testing } \\
\text { amoxicillin/clavulanate (8 } \\
\text { days) + levofloxacin (4 } \\
\text { weeks) }\end{array}$ & $\begin{array}{c}\text { Stable at } 18 \\
\text { months of follow- } \\
\text { up }\end{array}$ \\
\hline $\begin{array}{l}\text { Skelton W.P. } \\
\text { 4th; Taylor, Z.; } \\
\text { Hsu, J. }\end{array}$ & 2017 & Pyelonephritis & Urine & Cancer & $\begin{array}{l}73 \text { years/ } \\
\text { Female }\end{array}$ & $\begin{array}{l}\text { Initially cefalexin. } \\
\text { After antimicrobial } \\
\text { susceptibility testing } \\
\text { nitrofurantoin (7 days) }\end{array}$ & $?$ \\
\hline $\begin{array}{l}\text { Merino, R. E.; } \\
\text { Rebolledo 0.S; } \\
\text { Miquel, P.J. }\end{array}$ & 2017 & $\begin{array}{l}\text { Pancreatitis } \\
\text { Cholangitis }\end{array}$ & Blood & Rheumatic valvulopathy & $\begin{array}{l}55 \text { years/ } \\
\text { Female }\end{array}$ & Piperacillin/tazobactam & $?$ \\
\hline Tugcu, M. et al & 2017 & Pyelonephritis & Urine & $\begin{array}{l}\text { Urodynamic study, } \\
\text { peritoneal dialysis (PD) }\end{array}$ & $\begin{array}{l}57 \text { years/ } \\
\text { Male }\end{array}$ & Ceftriaxone (7 days) & $\begin{array}{l}\text { Stable at } 1 \\
\text { month of follow- } \\
\text { up }\end{array}$ \\
\hline Pan, Z. et al & 2017 & Pneumonia & Sputum & $\begin{array}{l}\text { Peritonitis, perforated } \\
\text { duodenal ulcer }\end{array}$ & $\begin{array}{l}74 \text { years/ } \\
\text { Male }\end{array}$ & $\begin{array}{l}\text { Initially piperacillin/ } \\
\text { tazobactam. After } \\
\text { exacerbation levofloxacin } \\
\text { + tigecycline (7 days) }\end{array}$ & $\begin{array}{c}\text { Stable at } 2 \\
\text { weeks of follow- } \\
\text { up }\end{array}$ \\
\hline Sia, C.S. et al & 2016 & Peritonitis & $\begin{array}{l}\text { Peritoneal } \\
\text { fluid }\end{array}$ & $\begin{array}{l}\text { Human immunodeficiency } \\
\text { vírus (CD4 122), chronic } \\
\text { kidney disease (CKD) }\end{array}$ & $\begin{array}{l}56 \text { years/ } \\
\text { Female }\end{array}$ & $\begin{array}{c}\text { Initially cefazoline }+ \\
\text { gentamicin ( } 6 \text { days). After } \\
\text { exacerbation ceftriaxone } \\
\text { (15 days) }\end{array}$ & $?$ \\
\hline $\begin{array}{l}\text { De Campos, } \\
\text { F.P; } \\
\text { Guimarães, } \\
\text { T.B.; Lovisolo, } \\
\text { S.M. }\end{array}$ & 2016 & $\begin{array}{l}\text { Pancreatic } \\
\text { pseudocyst }\end{array}$ & $\begin{array}{l}\text { Peritoneal } \\
\text { fluid }\end{array}$ & CKD, chronic pancreatitis & $\begin{array}{l}52 \text { years/ } \\
\text { Male }\end{array}$ & $?$ & Deceased \\
\hline Sitaula, S. et al & 2016 & Hepatic abscess & Pus & $\begin{array}{l}\text { DM, benign prostatic } \\
\text { hyperplasia }(\mathrm{BPH})\end{array}$ & $\begin{array}{l}62 \text { years/ } \\
\text { Male }\end{array}$ & $\begin{array}{l}\text { Initially piperacillin/ } \\
\text { tazobactam. After } \\
\text { antimicrobial } \\
\text { susceptibility testing } \\
\text { ceftriaxone (15 days) + } \\
\text { ciprofloxacin (28 days) } \\
\end{array}$ & $\begin{array}{c}\text { Stable at } 2 \\
\text { months of follow- } \\
\text { up }\end{array}$ \\
\hline
\end{tabular}




\begin{tabular}{|c|c|c|c|c|c|c|c|}
\hline Author & Year & $\begin{array}{c}\text { Clinical } \\
\text { manifestation }\end{array}$ & Culture site & Risk factors & Age/Gender & Treatment & Outcome \\
\hline $\begin{array}{l}\text { Gian, J.; } \\
\text { Cunha, B.A. }\end{array}$ & 2016 & Prostatitis & Urine & $\begin{array}{l}\mathrm{BPH}, \text { post transurethral } \\
\text { resection of the prostate }\end{array}$ & $\begin{array}{l}53 \text { years/ } \\
\text { Male }\end{array}$ & Fosfomycin (3 months) & $\begin{array}{c}\text { Stable at } 3 \\
\text { months of follow- } \\
\text { up }\end{array}$ \\
\hline $\begin{array}{l}\text { Tug'cu M., } \\
\text { et al. }\end{array}$ & 2016 & Pyelonephritis & Urine & PD, DM, urodynamic study & $\begin{array}{l}57 \text { years/ } \\
\text { Male }\end{array}$ & Ceftriaxone (7 days) & $\begin{array}{c}\text { Stable at } 1 \\
\text { month of follow- } \\
\text { up }\end{array}$ \\
\hline Cho, Y.J. et al & 2016 & Pneumonia & Sputum & Smoking & $\begin{array}{l}58 \text { years/ } \\
\text { Male }\end{array}$ & $\begin{array}{c}\text { Initially levofloxacilin + } \\
\text { piperacillin/tazobactam (7 } \\
\text { days). After antimicrobial } \\
\text { susceptibility testing, } \\
\text { piperacillin/tazobactam } \\
\text { (4 weeks) }\end{array}$ & $\begin{array}{c}\text { Stable at } 3 \\
\text { weeks of follow- } \\
\text { up }\end{array}$ \\
\hline $\begin{array}{l}\text { Vassalo, J. } \\
\text { et al }\end{array}$ & 2016 & Conjunctivitis & Pus & No relevant medical history & $\begin{array}{l}88 \text { years/ } \\
\text { Female }\end{array}$ & $\begin{array}{c}\text { Initially topical } \\
\text { gentamicin (8 days). } \\
\text { After exacerbation topical } \\
\text { ciprofloxacin (3 weeks) }\end{array}$ & $?$ \\
\hline $\begin{array}{l}\text { Vassalo, J. } \\
\text { et al }\end{array}$ & 2016 & Conjunctivitis & Pus & $?$ & $\begin{array}{l}71 \text { years/ } \\
\text { Male }\end{array}$ & Topical gentamicin & $?$ \\
\hline $\begin{array}{l}\text { Vassalo, J. } \\
\text { et al }\end{array}$ & 2016 & Conjunctivitis & Pus & $?$ & $\begin{array}{l}15 \text { years/ } \\
\text { Female }\end{array}$ & $?$ & $?$ \\
\hline $\begin{array}{l}\text { Vassalo, J. } \\
\text { et al }\end{array}$ & 2016 & Conjunctivitis & Pus & Postoperative & $\begin{array}{l}69 \text { years/ } \\
\text { Female }\end{array}$ & $?$ & $\begin{array}{c}\text { Recurrent } \\
\text { conjunctivitis }\end{array}$ \\
\hline Yoon, J.H. et al & 2015 & Cystitis & Urine & Cancer & $\begin{array}{l}16 \text { months/ } \\
\text { Male }\end{array}$ & $\begin{array}{l}\text { Initially cefotaxime + } \\
\text { ampicillin ( } 6 \text { days), then } \\
\text { cefpodoxime ( } 4 \text { days) }\end{array}$ & $\begin{array}{c}\text { Stable at } 2 \text { years } \\
\text { of follow-up }\end{array}$ \\
\hline Kim, S.W. et al & 2015 & Peritonitis & $\begin{array}{l}\text { Peritoneal } \\
\text { fluid }\end{array}$ & PD & $\begin{array}{l}65 \text { years/ } \\
\text { Male }\end{array}$ & $\begin{array}{l}\text { Initially cefazoline + } \\
\text { ceftazidime (6 days). } \\
\text { After antimicrobial } \\
\text { susceptibility testing, } \\
\text { ciprofloxacin + } \\
\text { ceftazidime (17 days) }\end{array}$ & $\begin{array}{c}\text { Stable at } 5 \\
\text { months of follow- } \\
\text { up }\end{array}$ \\
\hline $\begin{array}{l}\text { Gangcuangco, } \\
\text { L.M.; Saul, } \\
\text { Z.K. }\end{array}$ & 2015 & Cystitis & Urine & Dementia & $\begin{array}{l}92 \text { years/ } \\
\text { Female }\end{array}$ & Ceftriaxone (7 days) & $?$ \\
\hline $\begin{array}{l}\text { González- } \\
\text { González, L. } \\
\text { et al }\end{array}$ & 2015 & Bacteremia & Blood & $\mathrm{DM}$ & $\begin{array}{l}82 \text { years/ } \\
\text { Female }\end{array}$ & $\begin{array}{l}\text { Initially ceftriaxone. } \\
\text { After antimicrobial } \\
\text { susceptibility testing, } \\
\text { ciprofloxacin }\end{array}$ & $?$ \\
\hline$X u$, M. et al & 2015 & Pneumonia & Sputum & Cancer & $\begin{array}{l}60 \text { years/ } \\
\text { Male }\end{array}$ & Tigecycline + levofloxacin & Deceased \\
\hline $\begin{array}{l}\text { Lam, P.W.; } \\
\text { Salit, I.E. }\end{array}$ & 2014 & Bacteremia & Blood & $\begin{array}{l}\text { Cancer, PPI, seafood } \\
\text { consumption }\end{array}$ & $\begin{array}{l}56 \text { years/ } \\
\text { Female }\end{array}$ & $\begin{array}{l}\text { Initially ceftriaxone } \\
\text { + metronidazole. } \\
\text { After antimicrobial } \\
\text { susceptibility testing, } \\
\text { ceftriaxone (3 days) + } \\
\text { ciprofloxacin (7 days) }\end{array}$ & $?$ \\
\hline $\begin{array}{l}\text { Ershadi, A. } \\
\text { et al }\end{array}$ & 2014 & Cholecystitis & Biliary fluid & Alcoholic liver cirrhosis, DM & $\begin{array}{l}49 \text { years/ } \\
\text { Male }\end{array}$ & $\begin{array}{l}\text { Initially piperacillin/ } \\
\text { tazobactam + } \\
\text { vancomycin. Then } \\
\text { tigecycline (14 days) }\end{array}$ & $?$ \\
\hline $\begin{array}{l}\text { Zuberbuhler, } \\
\text { B.; Abedin, A.; } \\
\text { Roudsari, A. }\end{array}$ & 2014 & Conjunctivitis & Pus & $?$ & $\begin{array}{l}58 \text { years/ } \\
\text { Female }\end{array}$ & Topical chloramphenicol & $\begin{array}{c}\text { Stable at } 2 \\
\text { weeks of follow- } \\
\text { up }\end{array}$ \\
\hline $\begin{array}{l}\text { Koukoulalki, } \\
\text { M. et al }\end{array}$ & 2014 & Prostatitis & Urine & Transplant & $\begin{array}{l}67 \text { years/ } \\
\text { Male }\end{array}$ & Ciprofloxacin (4 weeks) & $\begin{array}{c}\text { Stable at } 6 \\
\text { months of follow- } \\
\text { up }\end{array}$ \\
\hline
\end{tabular}




\begin{tabular}{|c|c|c|c|c|c|c|c|}
\hline Author & Year & $\begin{array}{c}\text { Clinical } \\
\text { manifestation }\end{array}$ & Culture site & Risk factors & Age/Gender & Treatment & Outcome \\
\hline $\begin{array}{l}\text { Salmaggi, C. } \\
\text { et al }\end{array}$ & 2014 & Bacteremia & Blood & $\begin{array}{l}\text { Chronic obstructive } \\
\text { pulmonary disease, } \\
\text { bronchiectasis, cancer }\end{array}$ & $\begin{array}{l}70 \text { years/ } \\
\text { Male }\end{array}$ & $\begin{array}{l}\text { Initially ciprofloxacin } \\
\text { + metronidazole. } \\
\text { After antimicrobial } \\
\text { susceptibility testing, } \\
\text { ciprofloxacin (2 weeks) }\end{array}$ & $\begin{array}{l}\text { Stable at } 19 \text { days } \\
\text { of follow-up }\end{array}$ \\
\hline $\begin{array}{l}\text { Puerta- } \\
\text { Fernandez, S. } \\
\text { et al }\end{array}$ & 2013 & Bacteremia & Blood & $\begin{array}{c}\text { Pituitary adenoma (treated } \\
\text { with radiotherapy), BPH, fish } \\
\text { consumption }\end{array}$ & $\begin{array}{l}63 \text { years/ } \\
\text { Male }\end{array}$ & $\begin{array}{l}\text { Initially piperacillin/ } \\
\text { tazobactam. After } \\
\text { antimicrobial } \\
\text { susceptibility testing, } \\
\text { cefotaxime (10 days) }\end{array}$ & $?$ \\
\hline $\begin{array}{l}\text { Olson, D.S. } \\
\text { et al }\end{array}$ & 2013 & Pyelonephritis & Urine & $\begin{array}{l}\text { Coronary disease, heart } \\
\text { failure, CKD }\end{array}$ & $\begin{array}{l}89 \text { years/ } \\
\text { Male }\end{array}$ & $\begin{array}{l}\text { Initially ceftriaxone. } \\
\text { After antimicrobial } \\
\text { susceptibility testing, } \\
\text { ciprofloxacin (14 days) }\end{array}$ & $?$ \\
\hline Hu, A.Y. et al & 2012 & Bacteremia & Blood & Cancer, Post-ERCP & $\begin{array}{l}59 \text { years/ } \\
\text { Male }\end{array}$ & $\begin{array}{c}\text { Piperacillin/tazobactam } \\
\text { (14 days) }\end{array}$ & $?$ \\
\hline Teo, l. et al & 2012 & Cholecystitis & Biliary fluid & $\begin{array}{c}\text { Celiac disease, irritable } \\
\text { bowel syndrome, PPI }\end{array}$ & $\begin{array}{l}62 \text { years/ } \\
\text { Female }\end{array}$ & $\begin{array}{l}\text { Amoxicillin/clavulanate } \\
\text { (7 days) }\end{array}$ & $\begin{array}{l}\text { Stable at } 3 \\
\text { months of follow- } \\
\text { up }\end{array}$ \\
\hline Kim, S. H. et al & 2012 & $\begin{array}{l}\text { Necrotizing } \\
\text { fasciitis }\end{array}$ & Pus & Heart disease, DM, trauma & $\begin{array}{l}66 \text { years/ } \\
\text { Male }\end{array}$ & $\begin{array}{l}\text { Initially cefazolin } \\
\text { + clindamycin. } \\
\text { After antimicrobial } \\
\text { susceptibility testing, } \\
\text { ceftriaxone }\end{array}$ & $\begin{array}{c}\text { Stable at } 4 \\
\text { weeks of follow- } \\
\text { up }\end{array}$ \\
\hline Yokota, K. et al & 2012 & Bacteremia & Blood & Cancer & $\begin{array}{l}65 \text { years/ } \\
\text { Male }\end{array}$ & $\begin{array}{c}\text { Initially cefoperazone, } \\
\text { then meropenem + } \\
\text { piperacillin/tazobactam } \\
\text { (9 days) }\end{array}$ & $?$ \\
\hline $\begin{array}{l}\text { O'Connel, } \\
\text { K.; Kelly, J.; } \\
\text { Niriain, U. }\end{array}$ & 2010 & Cellulitis & Pus & Trauma & $\begin{array}{l}30 \text { years/ } \\
\text { Male }\end{array}$ & $\begin{array}{l}\text { Initially benzylpenicillin } \\
\text { + flucloxacillin } \\
\text { + clindamycin. } \\
\text { After antimicrobial } \\
\text { susceptibility testing, } \\
\text { ciprofloxacin added (2 } \\
\text { weeks) }\end{array}$ & $?$ \\
\hline $\begin{array}{l}\text { Alves, M.S.; } \\
\text { Riley, L. W.; } \\
\text { Moreira, B.M. }\end{array}$ & 2007 & Pancreatitis & $\begin{array}{l}\text { Peritoneal } \\
\text { fluid }\end{array}$ & Alcohol use disorder & $\begin{array}{l}45 \text { years/ } \\
\text { Male }\end{array}$ & $\begin{array}{c}\text { Initially ciprofloxacin + } \\
\text { metronidazole. Then } \\
\text { imipenem + amikacin } \\
\text { (15 days) }\end{array}$ & $?$ \\
\hline
\end{tabular}

DM - Diabetes mellitus; GERD - Gastroesophageal reflux disease; MRSA - Methicillin-resistant Staphylococcus aureus; PPI - Proton-pump inhibitor; ERCP - Endoscopic retrograde cholangiopancreatography; PD - Peritoneal dialysis; CKD - Chronic kidney disease; BPH - Benign prostatic hyperplasia

underlying risk factor is cancer ${ }^{1}$. Due to the rare occurrence of R. planticola infection in humans, most of the risk factors for this infection are largely deduced from published clinical cases. Immunosuppression, the use of proton pump inhibitors, comorbidities, exposure to soil or aquatic contaminants, invasive procedures, and seafood consumption (due to the ability to convert histidine to histamine) are thought to contribute to infection by R. planticola $a^{3,10-12}$. In addition, the most common primary sites of infection appear to be the biliary and urinary tracts ${ }^{1}$. R. planticola is generally sensitive to third generation cephalosporins, aminoglycosides, fluoroquinolones and carbapenems, but extensive resistance has been described, most commonly to ampicillin ${ }^{3}$ and piperacillin/tazobactam, with some cases of resistance to carbapenems and $\beta$-lactamases of broad spectrum reported ${ }^{7,13}$.

A comprehensive review of the literature identified 52 clinical cases published from 2007 to 2019 due to $R$. planticola infection in humans, selecting only case reports with full text (Table 1): four cases of pneumonia, one of endocarditis, twelve cases of urinary infection (six of cystitis, four of pyelonephritis and two of prostatitis), thirteen cases of intra-abdominal infection (one of cholangitis, three of cholecystitis, four of peritonitis, two of pancreatitis, one of appendicitis, one liver abscess and one pancreatic pseudocyst), two cases of skin and soft tissue infection (one due to necrotizing fasciitis and two due to cellulitis), thirteen cases of bacteraemia, four cases of conjunctivitis, one case of mucositis, one case of joint infection and one case of infection of the implantation site of a medical device. Within the 52 cases described, five patients died due to infectious complications, two of whom were diagnosed with R. planticola bacteraemia.

Only six of the documented cases occurred in patients under the age of 18 , with the average age of the reported cases being approximately 60 years and a higher prevalence in males. Approximately $60 \%$ of the patients had some degree of immunosuppression due to the underlying disease or treatments, increasing their susceptibility to 
opportunistic $R$. planticola infection. In five of the described cases, the risk factor for $R$. planticola infection seemed to be chronic use of PPI.

The majority of reported cases concern urinary tract infections, intra-abdominal infections and bacteraemia, with the number of the latter increasing in recent years. Our case adds another case of bacteraemia to those reported. As mentioned in the literature, the existence of comorbidities can contribute and facilitate infection by $R$. planticola. Our patient had several cardiovascular disease risk factors, chronic liver disease and was under phenytoin treatment, which can be linked to leukopenia, agranulocytosis and granulocytopenia. Regarding the alcohol withdrawal reported in our clinical case, chronic alcohol consumption can also play a capital role, due to cytokines imbalance and bone marrow shock, in increasing the susceptibility to infections, like $R$. planticola infection ${ }^{14}$. As far as we know, our patient had no consumption of shellfish or direct exposure to water or soil contaminants.

\section{CONCLUSIONS}

Due to the increasing prevalence and clinical relevance of $R$. planticola, an infection with this pathogen should be considered in patients with immunosuppression, subjected to invasive procedures, with chronic use of PPI, trauma or recent seafood consumption.

Currently, R. planticola remains largely susceptible to many antibiotics, but resistance to ampicillin, piperacillin/tazobactam and more recently carbapenems has been reported ${ }^{11}$. The antibiotic sensitivity test should be carried out in all cases to adequate the antibiotic therapy and decrease the likelihood of drug resistance.

\section{CONFLICTO DE INTERESES}

The author declares that there is no conflict of interest in this work.

\section{FINANCIACIÓN}

The author declares that there were no external sources of study for the performance of this article.

\section{ASPECTOS ÉTICOS}

The author declares that no data that allows identification of the patient appears in this article.

\section{REFERENCES}

1. Casarsa, C., Mearelli, F., Biolo, G. Severe Sepsis Due to Raoultella Planticola. American Journal of Infectious Disease. 2017;13(1):1-2.

2. González-González L.; Álvarez-Otero, J.; Ferreiro, J. L. L.; Aguado, J. F. Colangitis y bacteriemia por Raoultella planticola. Med Clin (Barc). 2015;144(5):231-232.

3. Tug 'cu M.; Ruhi, C.; Gokce, A. M.; Kara, M.; Aksarav, S. A case of urinary tract infection caused by Raoultella planticola after a urodynamic study. The Brazilian Journal of Infectious Diseases, 2017;21(2):196-198.

4. Ershadi, A.; Weiss, E.; Verduzco, E.; Chia, D.; Sadigh, M. Emerging pathogen: a case and review of Raoultella planticola. Infection. 2014;42(6):1043-6.

5. Gangcuangco, L. M.; Saul, Z. K. A novel case of Raoultella planticola urinary tract infection in a female: comment on 'Nosocomial pneumonia caused by carbapenem-resistant Raoultella planticola: a case report and literature review'. Infection. 2015;43(5):621-2.

6. Xu, M.; Xie, W.; Fu, Y.; Zhou, H.; Zhou, J. Nosocomial pneumonia caused by carbapenem-resistant Raoultella planticola: a case report and literature review. Infection. 2015;43(2):245-8.

7. Demiray, T.; Koroglu, M.; Ozbek, A.; Altindis, M. A rare cause of infection, Raoultella planticola: emerging threat and new reservoir for carbapenem resistance. Infection. 2016;44(6):713-717

8. Al-Sawaf, 0.; Garcia-Borreha, J.; J Vehreschild, J.; Thelen, P.; Fätkenheuer, G.; Shimabukuro-Vornhagen, A. et al. Pelvic cellulitis by Raoultella planticola in a neutropenic patient. J Infect Chemother. 2019;25(4):298-301.

9. González-González L.; Álvarez-Otero, J.; Ferreiro, J. L. L.; Aguado, J. F. Colangitis y bacteriemia por Raoultella planticola. Med Clin (Barc). 2015;144(5):231-232.

10. Mehmood, H.; Pervin, N.; Ul Haq, M. I.; Kamal, K. R.; Marwat, A.; Khan, M. A Rare Case of Raoultella planticola Urinary Tract Infection in a Patient With Immunoglobulin A Nephropathy. Journal of Investigative Medicine High Impact Case Reports. 2018 Jun.6:1-3. eCollection Jan-Dec 2018.

11. Subedi, R.; Dean, R.; Li, W.; Dhamoon, A. A novel case of Raoultella planticola osteomyelitis and epidural abscess. BMJ Case Rep. $2017 \mathrm{Jul}$ 13;2017:bcr2017220329

12. Westerveld, D.; Hussain, J.; Aljaafareh, A.; Ataya, A. A rare case of Raoultella planticolla pneumonia: an emerging pathogen. Respir Med Case Rep. 2017 Mar;21:69-70. eCollection 2017.

13. Cho, Y. J.; Jung, E. J.; Seong, J. S.; Woo, Y. M.; Jeong, B. J.; Kang, Y. M. et al. A case of pneumonia caused by Raoultella planticola. Tuberc Respir Dis (Seoul). 2016;79(1):42-5.

14. Povlow, M. R.; Carrizosa, J.; Jones, A. Raoultella planticola: Bacteriemia and sepsis in a patient with cirrhosis. Cureus. 2017;9(7):e1508. 